l'amélioration du lait pour l'alimentation infantile (en réalité, les globules de matière grasse en suspension dans le lait sont divisés et subdivisés $5,7,10$ fois..., améliorant physiquement l'aliment, en le rendant plus digestif et plus nutritif).

Les laits destinés à l'alimentation artificielle ou non spécifique du nourrisson doivent être supportés de façon satisfaisante. Pour cela, la température, le nombre journalier et la quantité des doses seront fonction des désirs de l'enfant, des modalités de l'allaitement naturel et des indications du pédiatre.

Personne ne peut avoir la prétention de remplacer l'alimentation spécifique de l'enfant (étant admis que la donneuse soit saine et le lait complet et correspondant), par un lait de vache modifié ; mais on peut le rapprocher chimiquement et l'adapter physiologiquement pour qu'il remplisse la fonction du succédané.

Si avec un lait modifié déterminé, on obtient un développement normal de l'enfant, le maintien de la santé et une bonne immunité, le résultat sera satisfaisant.

\title{
Analyse des laits maternisés
}

Elle est basée sur les mêmes règles que celles qui ont été mentionnées pour le lait normal et le lait en poudre.

\section{LES FACTEURS BACTÉRIOLYTIQUES ANTITUBERCULEUX DES FROMAGES (1)}

\author{
par
}

J. MEYER, J. TOUILLIER et J. MALGRAS

Les bacilles tuberculeux du type bovin introduits dans les fromages par le lait tuberculeux se maintiennent vivants et virulents dans les fromages mous jusqu'au stade de la décomposition putride finale, alors que ces germes disparaissent par un phénomène d'autostérilisation au bout dos quatre premières semaines dans les fromages à pâte dure.

Or, on pourrait incriminer trois facteurs dans ce processus :

$1^{\circ}$ Le lait étant chauffé dans les récipients de cuivre, les traces de ce métal entrant en contact avec le caillé pourraient être rendues responsables de la lyse des bacilles tuberculeux.

$2^{\circ}$ La fermentation lactique et les lactates jouent-ils leur rôle dans ce processus?

$3^{\circ}$ Les germes de la fermentation propionique existant dans la préparation des fromages cuits seraient-ils les agents de la destruction des bacilles tuberculeux ?

(1) C. R. Acad. Agric., 1952, 4, 167. 
Pour tenter de résoudre ce problème, nous avons tout d'abord examiné l'influence possible du cuivre sur les micro-organismes acidorésistants.

Nous avons pu nous rendre compte que le sulfate de cuivre ne possède aucun pouvoir bactéricide pour les concentrations correspondant aux conditions habituelles de fabrication des fromages d'Emmental et de Gruyère, puisque des doses de 0,20 à $0,25 \%$ n'inhibent pas la vitalité des bacilles acido-alcoolo-résistants.

\section{Action des ferments lactiques et des lactates}

Dans les cultures luxuriantes de bacilles acido-alcoolo-résistants, nous avons introduit des solutions de divers lactates pour arriver aux concentrations de $1 / 100,1 / 200,1 / 500,1 / 750,1 / 1.00,1 / 1.500$, $1 / 2.000,1 / 3.000,1 / 6.000$.

De plus, des cultures de bacilles lactiques ont été effectuées en milieu de Blickfeld. D'autre part, les bacilles acido-alcoolo-résistants ont été cultivés en milieu de J. Meyer.

Le développement optimum étant réalisé, on a introduit dans les cultures de mycobactéries les bacilles lactiques obtenus en milieu de Blickfeld.

De ces expériences nous pouvons conclure que les ferments lactiques et les lactates n'ont aucune influence défavorable sur la vitalité des bacilles acido-alcoolo-résistants.

\section{Action de la fermentation propionique}

Cette fermentation joue un rôle d'une importance capitale dans la fabrication des fromages cuits (maturation, caractères organoleptiques, formation des trous, etc.).

Nous avons effectué ces recherches au moyen de six séries de tubes de culture en milieu de jus de pomme de terre glycériné de J. Meyer. Ce substrat était ajusté à $p \mathbf{H} 7,2$; en présence du bacille propionique la réaction se stabilise vers le troisième jour à environ $p \mathrm{H} 5,0$. Nous avons pratiqué des contrôles de développement le huitième, resp. vingtième jour de culture selon les souches de bacilles acido-résistants employées. Pour les séries ayant été trouvées dépourvues de ces germes, nous en avons pratiqué à ce moment le réensemencement dans les conditions suivantes :

Deux séries de tubes à culture mixte initialement de BK + $\mathrm{BPr}$ ont été stérilisées par chauffage à $120^{\circ}$ pendant vingt minutes. L'une des séries a été employée sans modifier le $p \mathbf{H}(5,0)$, l'autre

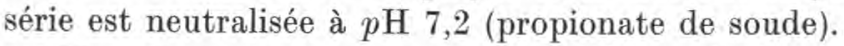

Nous exprimons ces expériences et leurs résultats dans le tableau suivant : 


\begin{tabular}{|c|c|c|c|c|c|c|c|}
\hline \multirow{3}{*}{\multicolumn{2}{|c|}{ Série de tubes }} & \multirow{3}{*}{ Culture } & \multicolumn{2}{|c|}{ Développement } & \multirow{2}{*}{\multicolumn{2}{|c|}{$\begin{array}{c}\text { Réensemencem } \\
\text { au BK après }\end{array}$}} & \multirow{3}{*}{$\begin{array}{l}\text { Résultat } \\
\text { après } 20 \mathrm{j} \\
\text { Dévelop } \\
\text { pement } \\
\text { du BK }\end{array}$} \\
\hline & & & \multirow[b]{2}{*}{ BK } & \multirow{2}{*}{$\mathrm{BPr}$} & & & \\
\hline & & & & & $\begin{array}{l}\text { Neutra- } \\
\text { lisation }\end{array}$ & $\begin{array}{c}\text { Stérili- } \\
\text { sation }\end{array}$ & \\
\hline 1 & . & Témoin BK & + & - & - & - & + \\
\hline 2 & $\ldots \ldots$ & Témoin $\mathrm{BPr}$ & - & + & - & - & - \\
\hline 3 & $\ldots \ldots \ldots \ldots$ & $\mathrm{BK}+\mathrm{BPr}$ & - & + & - & $\longrightarrow$ & - \\
\hline 4 & $\ldots \ldots \ldots \ldots \ldots$ & $\mathrm{BK}+\mathrm{BPr}$ & - & + & + & - & - \\
\hline 5 & $\ldots \ldots \ldots \ldots \ldots$ & $\mathrm{BK}+\mathrm{BPr}$ & 一 & + & - & + & + \\
\hline 6 & $\ldots \ldots \ldots \ldots \ldots$ & $\mathrm{BK}+\mathrm{BPr}$ & - & + & + & + & + \\
\hline
\end{tabular}

Des repiquages successifs devaient nous permettre de déterminer la limite de la vitalité des bacilles acido-résistants lors de leur contact avec les ferments propioniques.

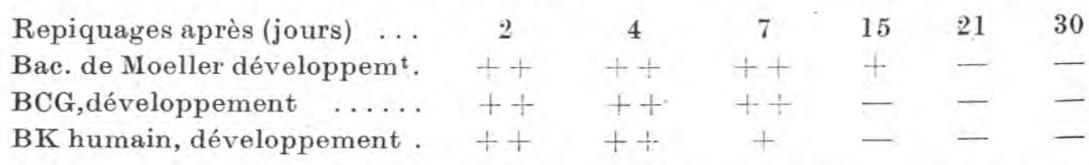

En étudiant ensuite le pouvoir bactéricide de divers propionates (Ca, Am., Al., Na., K, Cu, etc.) nous avons constaté que les sels d'aluminium et d'ammonium seulement détruisent les bacilles acidorésistants à des dilutions dépassant $1 / 2.000$, les autres propionates nécessitant des concentrations beaucoup plus fortes (1/200 à $1 / 750)$. Ce fait permet de conclure à l'effet antiseptique des cations d'aluminium et d'ammonium, alors que les anions propioniques semblent inactifs vis-à-vis des bactéries Ziehl + .

\section{Conclusions}

$1^{\circ}$ L'acidification des milieux par les bacilles propioniques ne suffit pas à expliquer l'inhibition de croissance des bacilles tuberculeux (tube 5).

$2^{\circ}$ Le propionate de sodium obtenu en neutralisant le milieu de culture n'empêche pas la croissance des bacilles acido-résistants (tube 6).

$3^{\circ}$ Par contre, dans les tubes où les bacilles acido-alcoolo-résistants se trouvent en contact avec le bacille propionique (tube 3 et tube 4), la croissance est inhibée et ceci sans distinction de la réaction du milieu (tube $3=p \mathrm{H}$ acide; tube $4=p \mathrm{H}$ neutre). En cońclusion, la présence du ferment propionique à l'état vivant dans un substrat occasionne l'arrêt du développement des bactéries acido-alcoolo-résistantes. 
$4^{0}$ Le bacille de la fléole a été lysé définitivement après un séjour de trois semaines dans les substrats à fermentation propionique. Ce même phénomène se produit déjà après deux semaines pour le BCG et le BK humain. Nos recherches expérimentales confirment une action bactéricide nette des ferments propioniques vivants sur les bactéries acido-alcoolo-résistantes.

(Service d'Hygiène et de Microbiologie de la Faculté de Pharmacie de Strasbourg.)

\section{BIBLIOGRAPHIE ANALYTIQUE}

\section{LES LIVRES}

Lembke (A.). - Mikroben in der Mich (Les microbes du lait). 1 livre relié toile de 189 pages. Editeur : Volkwirtschaftlicher Verlag G. m.b.H., Kempten (Allgäu). (Süddeutsche MolkereiZeitung). 1952. Prix : D.M. : 15,80.

Ce livre du savant Directeur de l'Institut de Bactériologie de l'Etablissement d'Essais et de Recherches de Laiterie de Kiel et de l'Institut de Recherches des Virus et de Médecine Expérimentale de Sielbeck (SchlesírigHolstein) est illustré par d'admirables mierophotographies des champignons inférieurs et des bactéries du lait prises pour la plupart à l'aide du microscope électronique. Le texte est digne des illustrations, car, sous une forme concise, il traite d'une façon magistrale la question de I'existence et de la croissance des mierobes du lait. De courts chapitres sont consacrés à la vie et aux travaux d'Antony Van Leeuwenhoek, d'Ernst Abbe, de Robert Koch et de Louis Pasteur.

C. Wolf.

\section{Demeter $(K . J$.$) . - Bakteriologische Untersuchungsmethoden$}

der Milchwirtschaft (Méthodes de recherches bactériologiques en laiterie). 1 liv. de 144 pages. $3^{\mathrm{e}}$ édition, revue et augmentée, avec 27 fig. Editeur : Eugen Ulmer, Stuttgart (z.Z. (14 a) Ludwigsburg), 1952. Prix : cartonné, D.M. 8,20 ; relié, D.M. 9,80 .

La troisième édition (revue et augmentée) de cet excellent livre vient de paraître. L'A. le $\mathrm{P}^{\mathrm{r}} K$. J. Nemeter, connait remarquablement les sujets qu'il traite et e'est pourquoi son livre est toujours accueilli avec le plus grand intérêt. Il est divisé en quatioe parties : lait et erème ; produits lactés; produits auxiliaires de la laiterio; matériaux de transport et d'emballage; contrôle des bâtiments et installations de la laiterie. En supplément, sont traités les examens des produits de désinfection et antibiotiques. Ce livre sera certainement utilisé avec profit par les institutions de recherches et d'essais de laiterie, par les grandes usines laitières, les étudiants, les hygiénistes et les bactériologues. Nous lui souhaitons le grand succès quilil mérite. 